\title{
Comparison of the mechanistic AGDISP ground boom spray model with experimental data
}

\author{
R.J. Connell ${ }^{1}$, W.C. Schou ${ }^{2}$, D. Nuyttens ${ }^{3}$, T.M. Wolf ${ }^{4}$ and J-P. Praat ${ }^{5}$ \\ ${ }^{1}$ Lincoln Ventures Ltd, PO Box 133, Lincoln, New Zealand \\ ${ }^{2}$ Scion, Private Bag 3020, Rotorua, New Zealand \\ ${ }^{3}$ Institute for Agricultural and Fisheries Research, Burg. Van Gansberghelaan 115, bus 1, \\ 9820 Merelbeke, Belgium \\ ${ }^{4}$ Agriculture and Agri-Food Canada, 107 Science Place, Saskatoon, Saskatchewan \\ S7N OX2, Canada \\ ${ }^{5}$ PA Handford and Assoc. Ltd, 104 Turere Lane, Te Awamutu, New Zealand \\ Corresponding author:Rob.connell@lvl.co.nz
}

\begin{abstract}
Since work reported in 2008, developments on the AGDISP ground boom model have improved results against New Zealand field trial drift data and also compare better with ground boom drift data sets from Belgium and Canada. The model still predicts more drift than the experimental data in most cases, especially for smaller droplet sizes, but does predict less drift for larger droplets at large distances downwind. The original AGDISP ground model was calibrated from Spray Drift Task Force data from the United States. The experimental methods need to be developed to improve collection efficiency and mass balance. The physics behind the model are discussed and proposed methods for improvement are suggested, including air velocities in the spray jet below the nozzle, the dispersion of the spray plume cloud at ground level due to atmospheric turbulence and wakes from the spraying equipment.
\end{abstract}

Keywords AGDISP, lagrangian, spray drift, ground boom, model, experimental data, mass balance.

\section{INTRODUCTION}

AGDISP uses Lagrangian theory to simulate the release of spray droplets into a turbulent velocity field and calculates the turbulent transport including evaporation onto the ground surface. Originally developed for aerial spraying (Bilanin et al. 1989), a ground boom option has been added (Teske et al. 2009). The model calculates ensemble average path from the nozzle for the range of droplet sizes and a Gaussian distribution around each average path.
Despite the model calibrating well with Spray Drift Task Force data from the United States the ground model did not calibrate well, overestimating drift with New Zealand data by 3.5 to 100 times (Woodward et al. 2008), Canadian data an average of 3.9 times (Connell et al. 2009), and Belgium data an average of 2.9 times for the flat fan data (R.B. Barton, unpublished data). Improvements to both the aerial and ground models have followed. Specific 
changes that impact the ground model are the improved evaporation of small droplets and the correction of a programming error that affected the magnitude of air entrainment generated by ground booms (Schou 2011). However, further development is required and this paper reports on comparisons of AGDISP 8.25 with field trial drift data from New Zealand (Zabkiewicz et al. 2008), Belgium (Nuyttens 2007; Nuyttens et al. 2007a, 2010, 2011) and Canada (Wolf \& Caldwell 2001) and discusses potential areas for improvement. Each of these trials used different methodologies as did the Spray Drift Task Force data used to calibrate AGDISP.

\section{MATERIALS AND METHODS}

The New Zealand trials (Woodward et al. 2008) reported four treatments using XR11003 and 04 flat fan nozzles at $150 \mathrm{kpa}$ and $300 \mathrm{kpa}$ at a target application rate of 200 litres/ha at $0.5 \mathrm{~m}$ above the ground of $0.1 \mathrm{~m}$ high grass with a ground speed of $2 \mathrm{~m} / \mathrm{s}$. One replicate of four $5 \mathrm{~m}$ wide, $20 \mathrm{~m}$ long swaths was carried out per treatment. Drift measurements were taken to $20 \mathrm{~m}$ downwind by collecting cations on a $3 \mathrm{~m}$ tape cut into three $1 \mathrm{~m}$ pieces and the values were averaged. The drop sizing was measured by laser diffraction using a Malvern laser (Stainier et al. 2006) in still air conditions.

The Belgian trials consisted of 108 spray runs with different spray application techniques. The trials included 32 reference runs across a wide range of weather conditions using Hardi ISO 11003 flat fan nozzles at $300 \mathrm{kpa}, 8 \mathrm{~km} / \mathrm{h}$ driving speed, $0.5 \mathrm{~m}$ boom height above the grass ( $0.6 \mathrm{~m}$ above soil), 180 litres/ha application rate, with one pass of a $27 \mathrm{~m}$ swath, $100 \mathrm{~m}$ in length. Data from three sets of collectors $10 \mathrm{~m}$ apart were averaged for each run or replicate. The fluorescent tracer was Brilliant Sulfo Flavine (BSF) and ground spray drift was collected to $20 \mathrm{~m}$ downwind using Machery Filter paper $0.25 \mathrm{~m}$ square with airborne drift measured at $5 \mathrm{~m}$ and $10 \mathrm{~m}$ up to a height of $5 \mathrm{~m}$ using pipe cleaners. Another 20 experiments varied all the main factors (nozzle type, size, boom height and driving speed) with three to six spray runs per experiment. Drop sizing was done using a Phase
Doppler Particle Analyser (PDPA) measuring system (Nuyttens et al. 2007b, 2009).

The 21 Canadian drift trials (Wolf \& Caldwell 2001) were carried out on $0.05 \mathrm{~m}$ to $0.1 \mathrm{~m}$ high desiccated wheat at $13 \mathrm{~km} / \mathrm{h}$ and $24 \mathrm{~km} / \mathrm{h}$ driving speeds, $0.6 \mathrm{~m}$ and $0.9 \mathrm{~m}$ spray heights respectively above the ground using AI1 10025 and 04, TT1 1005 and XR8003 flat fan nozzles at 275-445 kpa and 100 litres/ha application rate, with one spray pass with an $18 \mathrm{~m}$ wide swath, $150 \mathrm{~m}$ long. One replicate was done per trial with measurements using Rhodamine WT dye stabilised with 2,4-D on three lines, $10 \mathrm{~m}$ apart to $120 \mathrm{~m}$ downwind on the ground using Petri dishes and the airborne drift was measured with rotorods at $5 \mathrm{~m}$ downwind up to $2 \mathrm{~m}$ height. Droplet sizes were measured with Malvern laser for the AI nozzles to eliminate the effect of air inclusions in the droplets and PDPA for the other nozzles.

\section{RESULTS}

\section{Mass balance}

The most important factor in assessing experimental data for model development is mass balance. Mass balance provides guidance on the uncertainty of the data and its use in validating a model.

For the New Zealand experiments an average of $77.0 \%$ of the spray (ground only) was recovered by the cations (only ground deposition was measured; $75.3 \%$ on swath and $1.4 \%$ downwind to $20 \mathrm{~m}$ ).

The Belgian trials recovered an average of $78.5 \%$ with $76.7 \%$ on swath, and $1.8 \%$ drift downwind with $0.5 \%$ of this drift on the vertical collectors (up to $5 \mathrm{~m}$ height) at $5 \mathrm{~m}$ downwind.

The Canadian experiments recovered an average of $88.0 \%$, with $83.7 \%$ on swath and $4.3 \%$ drift downwind with $2.0 \%$ of this drift on vertical collectors (up to $2 \mathrm{~m}$ height) $5 \mathrm{~m}$ downwind. A photolysis study of the Canadian data showed that $5 \%$ of the Rhodamine dye was lost during the experimental procedure, which would account for a further $5 \%$.

\section{Ground drift deposition}

Comparisons of each set of field experiments with AGDISP v8.25 are shown in Figures 1 to 3. 
The New Zealand and Canadian data show averages for each nozzle type while the Belgian data are averages of 22 runs for the ISO 03 standard flat fan nozzles, and three runs for the 02, 04 and 06 standard flat fan nozzles. For two runs of the ISO 04 nozzles the wind direction was just over 30 degrees off perpendicular to the spray line, which is the maximum recommended angle to use AGDISP. AGDISP averaged 7.8 times the measured deposition of the New Zealand data, 1.5 times for the Canadian data and 2.2 times for the Belgian data.

\section{Vertical (airborne) drift flux}

Vertical flux was measured in both the Belgian (from 0.5 to $5 \mathrm{~m}$ ) and Canadian experiments (from $0.5 \mathrm{~m}$ to $2 \mathrm{~m}$ ) and two examples are shown in Figures $4 \mathrm{a} \& 4 \mathrm{~b}$.

Analysis of the total flux was undertaken by integrating the area of the two curves in Figure 4 from the height or (y) axis. The Belgian data (Figure 5a) showed AGDISP calculated an average of nearly 6 times the experimentally measured total flux for standard flat fan nozzles, while for Low Drift nozzles AGDISP estimated 1.6 times
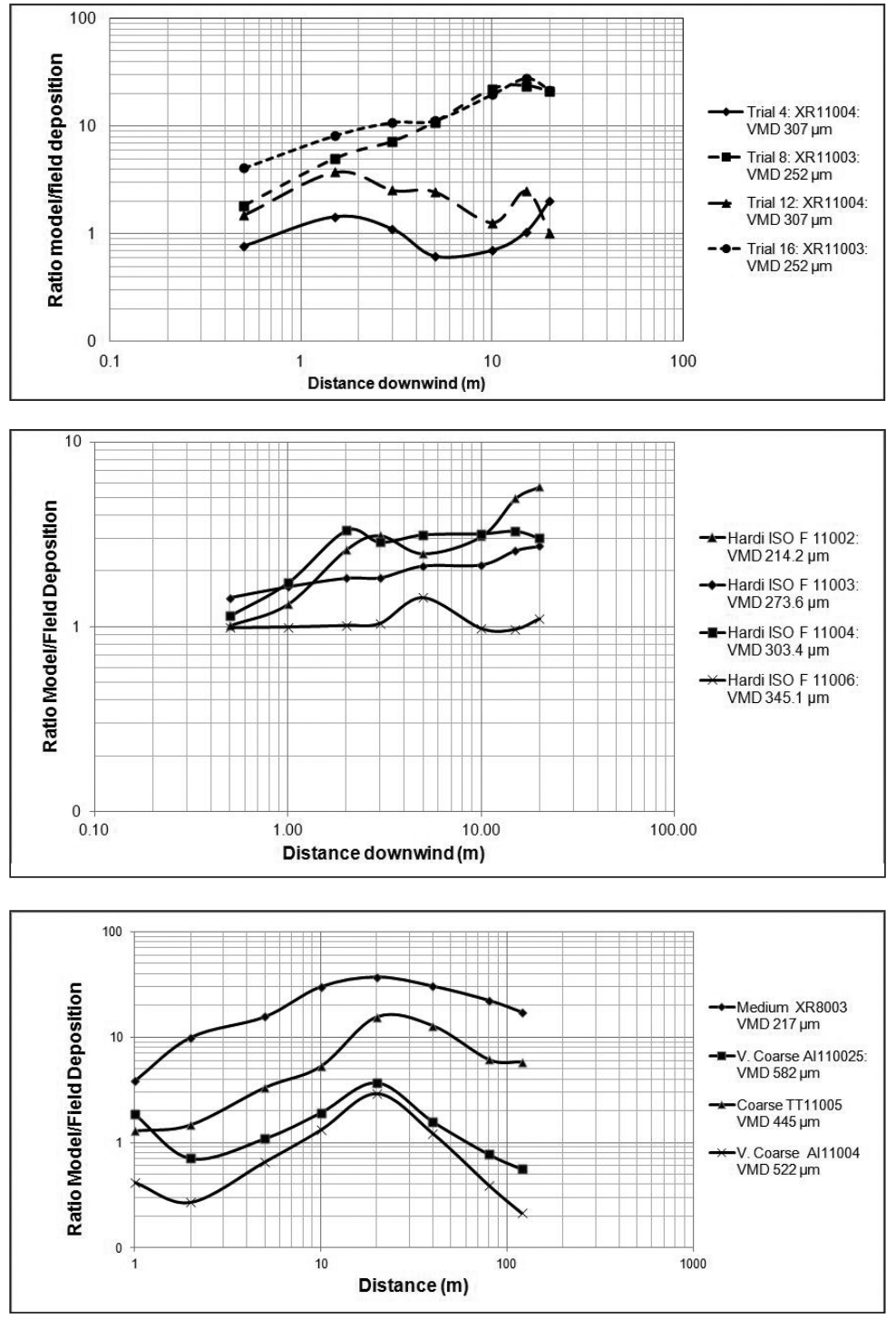

Figure 1 Deposition values presented as the ratio of modelled data to field data plotted against the distance downwind for the New Zealand trials.

Figure 2 Deposition values presented as the ratio of modelled data to field data plotted against the distance downwind for the Belgian trials.

Figure 3 Deposition values presented as the ratio of modelled data to field data plotted against the distance downwind for the Canadian trials. 
the measured total flux, and for the air injection nozzles AGDISP did not predict any vertical flux to $5 \mathrm{~m}$ so these data are not shown on Figure $5 \mathrm{a}$. For the Canadian data (assuming the flux rose to $4 \mathrm{~m}$ ) shown in Figure 5b, AGDISP calculated between 3 and 15 times the measured total flux for the XR8003 nozzles (Figure 5b), while for the
AI nozzles it calculated 0.2 to 0.6 of the measured total flux and 3 to 10 times the measured total flux for the TT11005 nozzles.

\section{Replicates}

When interpreting the results, the experimental design needs to be taken into consideration.

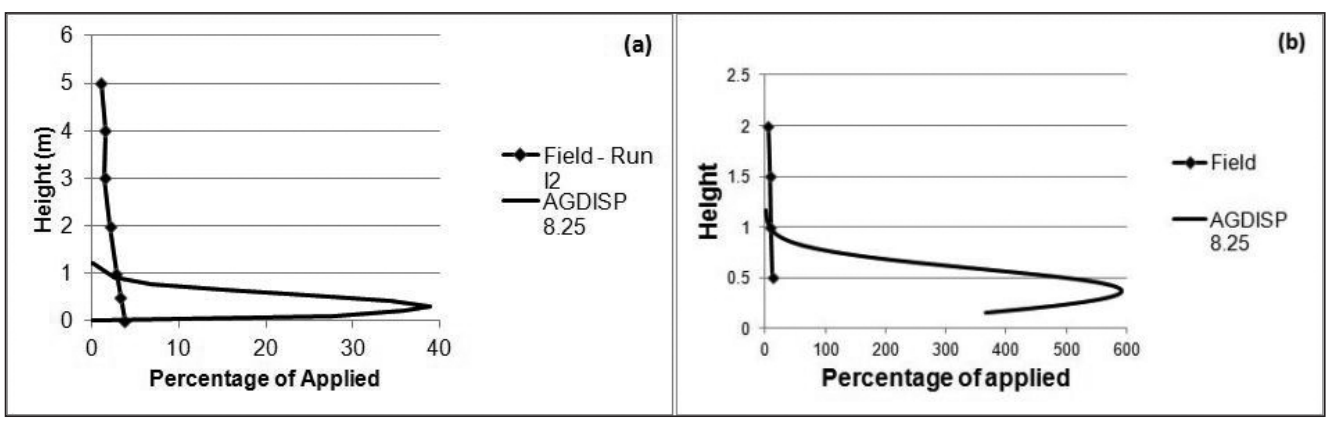

Figure 4 Vertical (airborne) drift flux measured and predicted using AGDISP 8.25 for (a) the Hardi ISO Flat Fan 11006 nozzle in the Belgian trials (run I2) and (b) the XR8003 nozzle in the Canadian field trials.

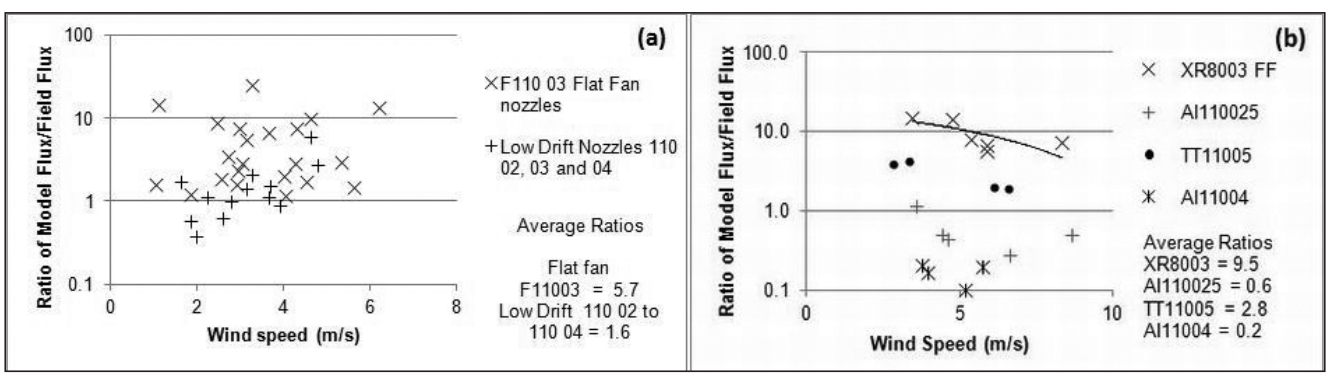

Figure 5 Ratio of model to field, of the total vertical flux at $5 \mathrm{~m}$ downwind, against wind speed for (a) Belgian trials using pipe cleaners and (b) Canadian trials using rotorods.

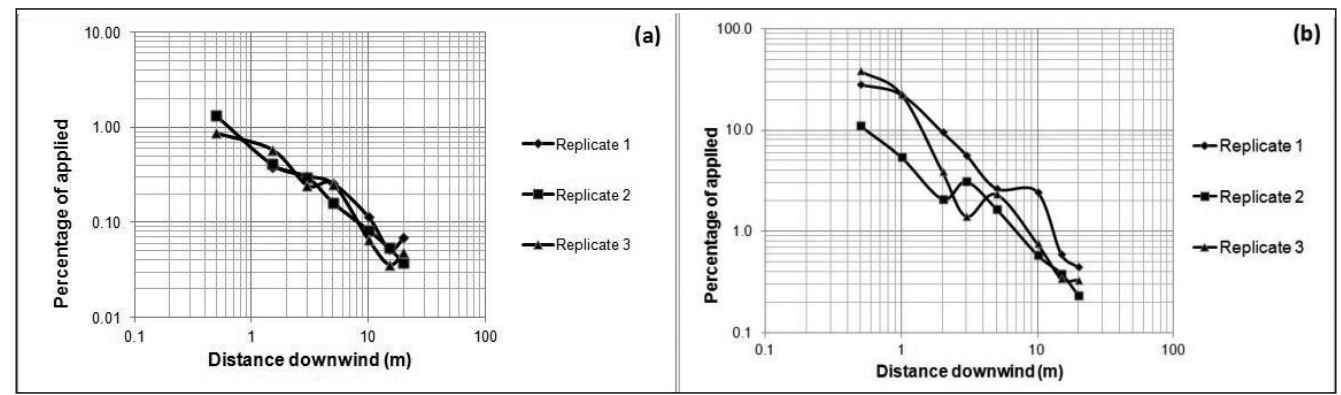

Figure 6 Deposition values presented as the percentage of applied versus distance downwind for (a) New Zealand data, XR11003 nozzle, VMD $252 \mu \mathrm{m}$ and (b) Belgian data, Hardy ISO F 11002 nozzle, VMD $214 \mu \mathrm{m}$. 
Replicates from the New Zealand trials shown in Figure $6 \mathrm{a}$ do not show the same degree of variation as replicates from the Belgian trials shown in Figure 6b.

\section{DISCUSSION}

The results of the studies show that $12 \%$ to $23 \%$ of the spray was not accounted for, especially in the Belgian and New Zealand studies. This means that it is difficult to fully evaluate the performance of the model. Is the model over-predicting or are the experiments under-collecting? To answer this question, the experimental method efficiency and the model physics both need to be investigated. Even when good agreement is found, accurately modelling an actual spray event will require further refinement to allow for the differences in the collection efficiencies of the experimental methods to those of the actual vegetation and ground surfaces onto which the spray will drift.

The ratio of model to measured decreased from an average of 12.4 (by reanalysis of these data) to 7.8 (to 63\%) for the New Zealand data (analysis of the results presented in Woodward et al. (2008)), while for the Canadian data the average ratio reduced from 3.9 to 1.5 (to $38 \%$ ) and the Belgian data from 2.9 to 2.2 (to $76 \%$ ).

All the graphs of the results (Figures 1-3) show that the ratio of model/field result increased in value for smaller droplets. The New Zealand and Belgian data showed an increase in this ratio with distance downwind. Both trials measured deposition to $20 \mathrm{~m}$. The Canadian data also showed this trend to $20 \mathrm{~m}$ downwind but further downwind the ratio reduced to less than the measured data, which could be the result of less wake effects from the sprayer further downwind.

The New Zealand ground drift data had a higher model/field ratio for the 03 nozzles compared to the Belgian data, while the Canadian data had an even higher ratio. The reason for the increase in ratios could be the droplet sizes, measured at $273 \mu \mathrm{m}$ for the Belgian nozzles, $252 \mu \mathrm{m}$ for the New Zealand nozzles and $217 \mu \mathrm{m}$ for the Canadian nozzles. The very high Canadian ratio could also be the faster driving speed and larger apparatus, as the AGDISP model does not take this factor into account at present. The ground boom model uses a description of turbulence using the shear velocity and does not consider wakes from a sprayer and the boom, which could affect the results. The change in turbulence from the different wakes could be characterised in libraries for each sprayer generated using computational fluid dynamic models (CFD online 2012).

Figure $6 \mathrm{~b}$ highlights the large variation in deposition between two independent repetitions, as replicates 1 and 3 have almost the same wind speed, temperature and humidity but still show more variability than the replicates in Figure 6 a. The replicates shown in Figure $6 \mathrm{a}$ are 0.5 seconds apart ( $1 \mathrm{~m}$ separation of the tapes and a driving speed of $2 \mathrm{~m} / \mathrm{s}$ ). A typical timescale for turbulence in the wind direction close to the ground is 5 seconds (Yahaya et al. 2003), and the lateral time scale will be about 40 to $50 \%$ of this, from data in Counihan (1975), or about 2 seconds. This means the replicates in Figure 6a are definitely dependent, so they should be called quasi-replicates.

It has been recommended that up to 20 replicates are needed under very similar weather conditions to provide a representative sample (H.W. Thistle, USDA Forest Service, USA, personal communication). Examining the inflowing air turbulence characteristics upwind of the sprayer may reveal that fewer replicates are necessary to give a representative sample.

The experimental results in Figure 4 show that vertical flux measured extends much higher than that calculated by AGDISP. The mechanism used in the model needs to be evaluated and improved especially to consider the wakes from the sprayer and boom.

The model is underpredicting vertical fluxes for Air Induction nozzles, which could be a result of different properties, such as the droplets being less dense, and differing evaporation and breakup characteristics and droplet size measurement, all which need to be investigated.

Analysis of the total vertical fluxes for Flat fan nozzles showed AGDISP could be over-predicting but equally the experimental collection may not be efficient. The collection efficiency of the pipe cleaners for the Belgian study has not been 
evaluated. A possible reason for the Canadian data having less efficient collection was that the rotorods may have lower collection efficiency at higher wind speeds especially at wind speeds of $9.0 \mathrm{~m} / \mathrm{s}$, close to the rotorod speed of $10 \mathrm{~m} / \mathrm{s}$ (May et al. 1976). However, Figure 5b indicates that experimental data and model prediction became closer with increasing wind speed, which seems to contradict the previous statement. For the pipe cleaners there was no dependence on wind speed (Figure 5a) for the flat fan nozzles, but for the low drift nozzles the ratio increased with increasing wind speed, which suggests that collection method is influencing the results. The May et al. (1976) paper evaluated collection efficiencies of rotorods for droplets up to $50 \mu \mathrm{m}$, and this was less than the theoretical efficiency using May \& Clifford (1967). The AGDISP model showed droplets up to $100 \mu \mathrm{m}$ are present $5 \mathrm{~m}$ downwind, for which rotorod field collection efficiencies are not known. Further work on the collection efficiencies of both methods is necessary to fully evaluate the experimental measurements.

The air velocities in the jet below the nozzle measured by Miller et al. (1996) are $2 \mathrm{~m} / \mathrm{s}$ at $0.5 \mathrm{~m}$ below a F110015 nozzle. This velocity using formula (2) of Teske et al. (2009) and the given parameters is $10 \mathrm{~m} / \mathrm{s}$ for the flat fan nozzles, which is well above the velocity measured by Miller et al. (1996). If the values inserted into formula (2) for the Canadian data are used, then this velocity drops to $1.75 \mathrm{~m} / \mathrm{s}$. The air velocities in the jet (velocities of droplets less than $40 \mu \mathrm{m}$ as these very small sized droplets adjust very quickly to the air velocity around them) $0.5 \mathrm{~m}$ below the nozzle measured by Nuyttens (2007) were about $1.75 \mathrm{~m} / \mathrm{s}$ for standard flat Fan nozzles and $0.7-1.2 \mathrm{~m} / \mathrm{s}$ for AI nozzles. Improvements to this calculation, will allow better determination of the distance below a nozzle when a given sized droplet leaves the jet and begins to drift downwind.

\section{CONCLUSION}

The new version of AGDISP gives improved deposition for standard flat fan nozzles that are generally higher than experimental data, with the ratio increasing for smaller droplet sizes. For AI nozzles AGDISP results are higher than experimental data, and ongoing research is needed to include the properties of AI droplets in AGDISP. There is still considerable spray unaccounted for in the experiments, and further work to improve and assess the collection efficiency is needed for all the techniques. The AGDISP model still needs further development in other areas, including the incorporation of the correct air velocity in the jet below the nozzle, the distance at which a given sized droplet leaves the jet and the atmospheric dispersion at ground level. These are fundamental aspects of a ground model, and are also a requirement of any orchard model, making improvements in this area paramount.

\section{ACKNOWLEDGEMENTS}

This work was undertaken as part of MSI contract LVLX0901, 'Protecting NZ's environment from pesticide exposure'. Thank you to Andrew Hewitt for providing Tom Wolf's data and Rory Barton for undertaking 2010 analysis of the Belgian data.

\section{REFERENCES}

AGDISP 8.25 2011. USDA Forest Service Spray Modelling Software, version 8.25. Harold W. Thistle, USDA Forest Service, 180 Cranford Street, Morgantown, WV, 26505, USA.

Bilanin AJ, Teske ME, Barry JW, Ekblad RB 1989. AGDISP: The aircraft spray dispersion model, code development and experimental validation. Transactions of the American Society of Agricultural Engineers 32(1): 327334.

CFD Online 2012. http://www.cfd-online.com (accessed 15 April 2012).

Connell RJ, Hewitt AJ, Wolf T, Miller PCH 2009. WTDISP, Adapting a Lagrangian ground sprayer model using wind tunnel data. $18^{\text {th }}$ World IMACS/MODSIM Congress, 13-17 July 2009, Cairns, Australia. Pp. 4402-4408.

Counihan J 1975. Adiabatic atmospheric boundary layers: A review and analysis of data from the period 1880 to 1972 . Atmospheric Environment 9: 871-905.

May KR, Clifford R 1967. The impaction of aerosol particles on cylinders, spheres, ribbons and discs. Annals of Occupational Hygiene 10: 83-95. 
May KR, Pomeroy NP, Hibbs S 1976. Sampling techniques for large windborne particles. Journal of Aerosol Science 7: 53-62.

Miller PCH, Butler Ellis MC, Tuck CR 1996. Entrained air and droplet velocities produced by agricultural flat-fan nozzles. Atomization and Sprays 6: 693-707.

Nuyttens D 2007. Drift from field crop sprayers: The influence of spray application technology determined using indirect and direct drift assessment means. PhD thesis, Katholieke Universiteit Leuven, Leuven, Belgium. 267 p.

Nuyttens D, De Schampheleire M, Baetens K, Sonck B 2007a. The influence of operator controlled variables on spray drift from field crop sprayers. Transactions of the ASABE 50: 1129-1140.

Nuyttens D, Baetens K, De Schampheleire M, Sonck B 2007b. Effect of nozzle type, size and pressure on spray droplet characteristics. Biosystems Engineering 97: 333-345.

Nuyttens D, De Schampheleire M, Verboven P, Brusselman E, Dekeyser D 2009. Droplet sizevelocity characteristics of agricultural sprays. Transactions of the ASABE 52: 1471-1480.

Nuyttens D, De Schampheleire M, Verboven P, Sonck B 2010. Comparison between indirect and direct spray drift assessment methods. Biosystems Engineering 105: 2-12.

Nuyttens D, De Schampheleire M, Baetens K, Brusselman E, Dekeyser D, Verboven P 2011. Drift from Field Crop Sprayers using an Integrated Approach: Results of a 5 Year Study. Transactions of the ASABE 54: 403-408.

Schou WC 2011. AGDISP Model Developments. Pesticide Application and Drift Modelling Workshop, 29-31 August 2011, Lincoln University, New Zealand.
Stainier C, Destain M-F, Schiffers B, Lebeau F 2006. Droplet size spectra and drift effect of two phenmedipham formulations and four adjuvant mixtures. Crop Protection 25: 12381243.

Teske ME, Miller PCH, Thistle HW, Birchfield NB 2009. Initial development and validation of a mechanistic spray drift model for ground boom sprayers. Transactions of the ASABE 52: 1089-1097.

Wolf TM, Caldwell BC 2001. Development of a Canadian spray drift model for the determination of buffer zone distances. In: Bernier D, Campbell DRA, Cloutier D ed. Expert Committee on Weeds - Comité d'Experts en Malherbologie, Proceedings of the 2001 National Meeting, Québec City. Sainte-Anne-de-Bellevue, Québec, Canada. p 60 .

Woodward SJR, Connell RJ, Zabkiewicz JA, Steele KD, Praat JP 2008. Evaluation of the AGDISP ground boom spray drift model. New Zealand Plant Protection 61: 164-168.

Yahaya S, Frangi JP, Richard DC 2003. Turbulence characteristics of a semiarid atmospheric surface layer from cup anemometers - effects of soil tillage treatment (Northern Spain). Annales Geophysicae 21: 2119-2131.

Zabkiewisz JA, Steele KD, Praat JP 2008. Determination of spray drift using multiple metal cations as tracers. New Zealand Plant Protection 61: 159-163. 